\title{
A Smart Mobile Lab-on-Chip-Based Medical Diagnostics System Architecture Designed For Evolvability
}

Patou, François; Dimaki, Maria; Svendsen, Winnie Edith; Kjærgaard, Claus; Madsen, Jan

Published in:

Proceedings of the Euromicro Conference on Digital System Design 2015

Link to article, DOI:

10.1109/DSD.2015.11

Publication date:

2015

Document Version

Peer reviewed version

Link back to DTU Orbit

Citation $(A P A)$ :

Patou, F., Dimaki, M., Svendsen, W. E., Kjærgaard, C., \& Madsen, J. (2015). A Smart Mobile Lab-on-ChipBased Medical Diagnostics System Architecture Designed For Evolvability. In Proceedings of the Euromicro Conference on Digital System Design 2015 (pp. 390-398). IEEE. https://doi.org/10.1109/DSD.2015.11

\section{General rights}

Copyright and moral rights for the publications made accessible in the public portal are retained by the authors and/or other copyright owners and it is a condition of accessing publications that users recognise and abide by the legal requirements associated with these rights.

- Users may download and print one copy of any publication from the public portal for the purpose of private study or research.

- You may not further distribute the material or use it for any profit-making activity or commercial gain

- You may freely distribute the URL identifying the publication in the public portal 


\section{A Smart Mobile Lab-on-Chip-Based Medical Diagnostics System Architecture Designed For Evolvability}

\author{
François Patou, \\ Maria Dimaki, Winnie E. Svendsen \\ dept. of Micro- and Nanotechology \\ Tech. Univ. of Denmark, DTU \\ Lyngby, Denmark \\ email:frpato@nanotech.dtu.dk
}

\author{
Claus Kjægaard \\ dept. of Electrical Engineering \\ Tech. Univ. of Denmark, DTU \\ Lyngby, Denmark \\ email: clkj@dtu.dk
}

\author{
Jan Madsen \\ dept. of Applied Mathematics \\ and Computer Science \\ Tech. Univ. of Denmark, DTU \\ Lyngby, Denmark \\ email:jama@dtu.dk
}

\begin{abstract}
Unprecedented knowledge levels in life sciences along with technological advances in micro- and nanotechnologies and microfluidics have recently conditioned the advent of Lab-on-Chip (LoC) devices for In-Vitro Medical Testing (IVMT). Combined with smart-mobile technologies, LoCs are pervasively giving rise to opportunities to better diagnose disease, predict and monitor personalised treatment efficacy, or provide healthcare decision-making support at the Pointof-Care (PoC). Although made increasingly available to the consumer market, the adoption of LoC-based PoC In-Vitro Medical Testing (IVMT) systems is still in its infancy. This attrition partly pertains to the intricacy of designing and developing complex systems, destined to be used sporadically, in a fast-pace evolving technological paradigm. System evolvability is therefore key in the design process and constitutes the main motivation for this work.

We introduce a smart-mobile and LoC-based system architecture designed for evolvability. By propagating LoC programmability, instrumentation, and control tools to the highlevel abstraction smart-mobile software layer, our architecture facilitates the realisation of new use-cases and the accommodation for incremental LoC-technology developments. We demonstrate these features with an implementation allowing the interfacing of LoCs embedding current- or impedancebased biosensors such as Silicon Nanowire Field Effect Transistors (SiNW-FETs) or electrochemical transducers. Structural modifications of these LoCs or changes in their specific operation may be addressed by the sole reengineering of the mobilesoftware layer, minimising system upgrade development and validation costs and efforts.
\end{abstract}

Keywords-Lab-on-Chip; Smartphone; Point-of-Care; InVitro Medical Diagnostics; System Evolvability; System Architecting;

\section{INTRODUCTION}

A. The healthcare landscape and Point-of-Care in-vitro medical testing (IVMT)

The healthcare landscape of our modern societies is rapidly changing. The increasing healthcare demand of the Western World's aging population stretches the restricted budgets of our institutions and challenges the limited availability of our care personnel and facilities. Chronic diseases hold a particular place in that scenery. By accounting to up to $84 \%$ of the annual 3 trillion dollars of healthcare expenditures in the United States [1], conditions like cancer, heart-failure or neurodegenerative diseases are not only debilitating but also represent a significant economic burden, threatening health accessibility and quality of care. In the developing world, infectious diseases like HIV/AIDS or tuberculosis remain a predominant cause of death mainly on the account of insufficient resources and infrastructures [2], [3].

Despite these worrisome observations, unprecedented knowledge levels in life science, especially in the -omics disciplines (genomics, transcriptomics, proteomics, etc. [4]) and recent technological advances are radically changing both the way and efficacy with which we diagnose and tackle disease. Medical diagnostics, therapeutics and clinical management are all being remodelled in an attempt to personalise medicine and empower patients with tools to become prominent contributors of their own health management. [1], [5].

More specifically, in-vitro medical testing (IVMT) plays a preponderant role in the identification of disease, helping the initiation of appropriate therapy and allowing the further monitoring of treatment outcome. Initially driven by a centralized, technical, high-throughput biological sample analysis process, the quantification of analytes in biological samples is undergoing a major paradigm shift, transitioning to a diagnostician and patient-oriented Point-of-Care (PoC) decision-making support system. This revolution has been made possible by advances in nanotechnology, microfluidics, and biosensing, which combined, have conditioned the advent of Lab-on-Chip (LoC) technologies and systems. LoCs are designed to embed most of the functionalities of a central chemical and biological analyses laboratory on chips with footprints rarely larger than a few centimetres square. They allow for routines tests to be performed using sample volumes down to a few nanoliters. LoC techniques enable PoC in-vitro testing, potentially empowering diagnosticians and patients to carry out automated screening, diagnostics or monitoring procedures outside of the clinical setting. 


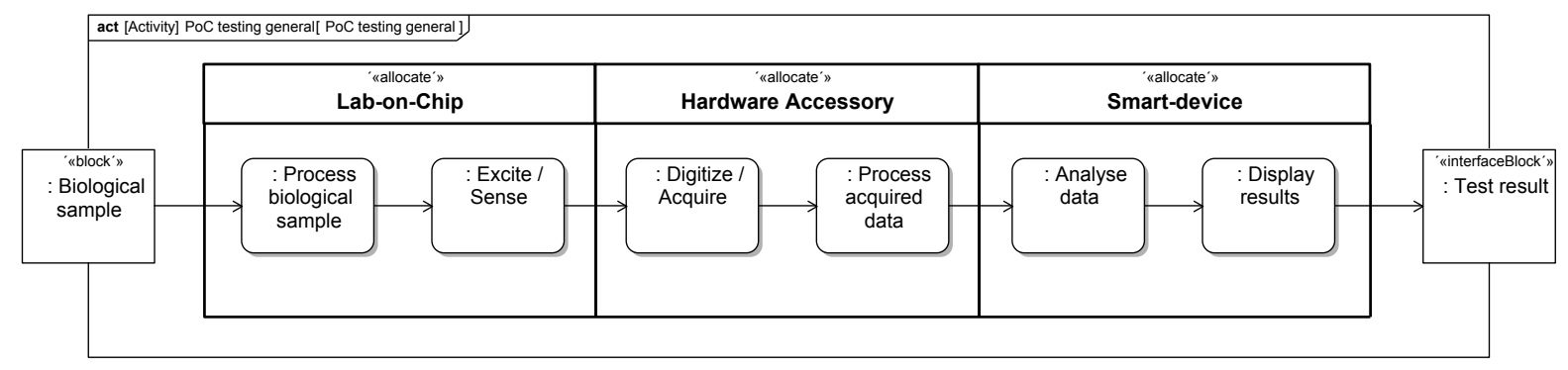

Figure 1. LoC-based PoC smart-mobile IVMT concept. In simplified cases, if no fluid actuation is required (e.g. for glucose monitoring), the analytical process starts from the LoC with the biological sample making its way to the LoC biosensor, the HWA then handles signal acquisition and processing before transmitting the results to the smart-device. Simple analytics such as mathematical fitting to a calibration curve is either handled in the HWA or the smart-device before results are fed back to the end-user.

LoC devices ubiquitously rely on microfluidic technology to carry out on-chip processing of the biological sample (e.g. blood, saliva, etc.). Some of the most common microfluidic functions include the separation, pre-concentration, and isolation of the analytes of interest [6]-[8]. The operation of specific biosensing schemes enables the extraction of the relevant predictive information. Detection methods relying on optical [9], electrochemical [10], or impedimetric phenomema [11] are examples of the wide spectrum of technologies that can be leveraged in applications ranging from the determination of immunoprotein biomarkers concentrations [12] to cell sorting [13] or rare cell isolation [14], and to single-cell [15] or single-molecule analysis [16]. Benefiting from recent progress in solid-state nanopores, -omics-sequencing techniques have emerged in the $\mathrm{PoC}$ paradigm [17]. Drastic reductions in cost-per sequenced base-pair [18] along with progresses in -omics big-data analytics [19] are promising a new range of applications to make their way to consumer-products. Cancer molecular diagnostics but also pathogen identification or drug-response profiling are just a few examples [4].

The complexity of LoC systems can be substantial, especially when flow actuation for biological sample processing is instrumented such as for digital- [20] or droplet-based [21] microfluidic systems or when addressing complex sensing mechanisms (e.g. fluorescence, mass spectrometry, etc.). Instrumentation complexity (e.g. hardware, optics, control algorithms, etc.) and costs have been arguably considered a limitation to the adoption of advanced LoCs in PoC settings, notably in the developing world [22].

\section{B. IMVT and smart-mobile technology}

Another key factor in recent patient-empowerment and the remodelling of our healthcare landscape is the advent of smart mobile technology. Smart-phones and tablets are already providing widely adopted mobile-health software solutions, ranging from image-analysis-based dermatologic evaluation [23] to applications helping patients to better adhere to their pharmacological treatment [24]. More recently, numerous physical activity tracking applications have emerged from the upcoming of smart-sensors and wearables, allowing the monitoring of basic health-metrics such as heart rate, blood pressure, or body-mass index. These biological signals are known to strongly correlate with disease severity for conditions such as hypertension or cardiac arrhythmias. They represent in those cases valuable surrogate biomarkers and validated clinical endpoints that can be evaluated to assess treatment efficacy or prognosis.

These applications illustrate how smart-devices can be leveraged for their peripherals, wireless interfaces, computational power, software flexibility, cloud connectivity, or simply for the possibilities they offer in terms of user-experience. These features constitute as many opportunities in attempting to cope with the limitations of instrumented-LoC technologies in a PoC context. Smart-mobile technology can indeed be used to address complex sample handling flow actuation [25] or to interface elaborate sensing technologies for quantitative analyses. Some notable recent successes include the reliable PoC diagnosis of HIV and syphilis in less than 15 minutes in a low-income country using a LoC-dongle [26], [27]. Although this example represents a milestone in the upcoming advent of such systems, their adoption today remains scarce, with the notable exception of glucose monitoring and of a few general inflammation and hormonal biomarkers [28].

Pertaining to this slow democratisation, it has been argued that the instrument and consumable business model is, in the consumer market, limited by low frequency of use [29]. If unlike glucose-monitoring, IVMT tests need only be performed sporadically, high average cost-per-test is likely to result from the initial investment for the instrument and from the low-volume, and therefore pricy consumables (LoCs). This limitation is substantial and raises the critical question of what specific design issues need to be addressed in order to ensure that the development, validation and commercialisation of these systems can be profitable and thus to guarantee their societal impact. 


\section{LOC-BASED POC IVMT SYSTEMS AND EVOLVABILITY}

\section{A. Evolvability as a design requirement}

While more than 150.000 scientific publications discuss the predictive values of thousands of potential biomarkers for disease diagnosis or therapy effectiveness assessment, only about a hundred are currently validated for use in clinical practice [30]. Our increasing understanding of disease mechanisms will yet much likely result in the validation of other candidate molecules [31]. Concurrently, technological developments in LoC technologies (e.g. microfluidics, biosensing, etc.), advanced computational tools for LoC design and operation [32] and progress in -omics-based predictive analytics will give rise to new potential LoCbased PoC IVMT applications and help achieve greater systems performances. Leveraging these opportunities will yet necessitate addressing greater system complexity. Blankcanvas full system design will steadily translate into higher implementation and validation costs and efforts, along with greater risks associated with longer time-to-market. Complex LoC-based PoC IVMT will thus increasingly benefit from system evolvability.

System evolvability can be defined as "the capacity of a system to successfully adapt to changing requirements throughout its lifecycle without compromising architectural integrity. [...] meet the new needs of the customer in a more cost effective manner than developing a new system" [33]. System evolvability should minimise the costs and efforts associated with system re-design and with development iterations of hardware, software and interfaces. It will facilitate system maintainability and promote system legacy, the latter of which we identified as an important factor for success in a low frequency-of-use instrument/consumable business model.

\section{B. Smart-device and LoC-based system evolvability criteria}

LoC devices were originally application-specific (i.e. operated in a fixed manner to realise a given use-case: biological sample processing and data acquisition schemes were mostly determined at the LoC design-stage). Benefiting to LoC-based systems evolvability, configurable or programmable LoCs have recently emerged [34], [35]. They enable the realisation of custom biological sample processing functions or the characterisation of chosen fluid or analytes properties depending on the use-case of interest. They require to that end the generation of specific LoC programs, determining the actuation (e.g. via micro-valves, pumps or electrowetting) and acquisition schemes (e.g. sensor excitation and readout) necessary to realise these functions. The increasing complexity of reprogrammable LoC devices puts stringent requirements on LoC control and instrumentation hardware and software. Programming and program execution handling tools are necessary in order to

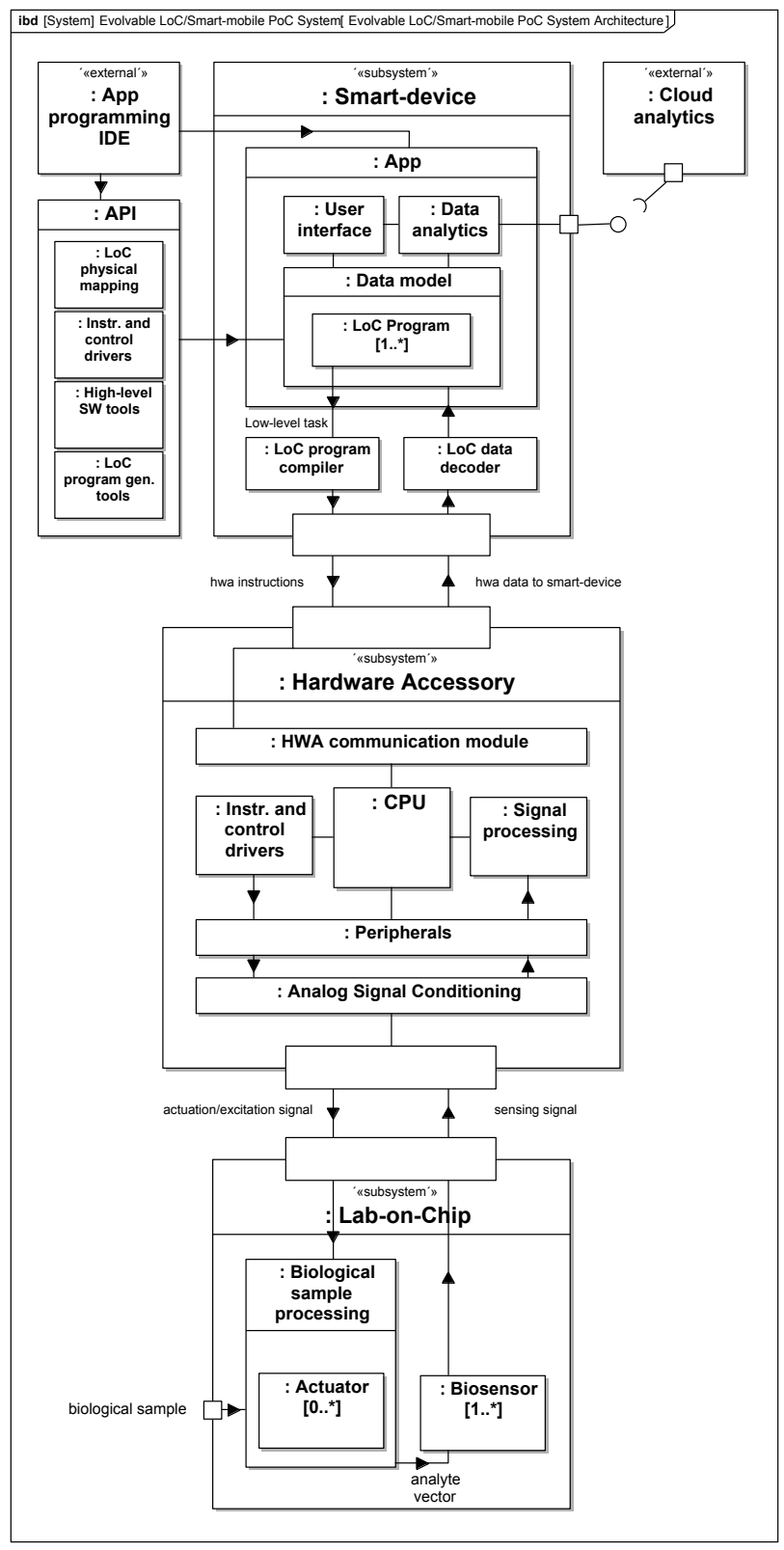

Figure 2. Evolvable system architecture diagram - A mobile-software API allows for the generation of custom pre-compiled or run-time LoC programs to be integrated in an application's data model. Low-level tasks, relying on the API instrumentation and control drivers are translated to HWA/LoC instructions through a compiler object (left part of the diagram). Low-level task execution is handled at the mobile-software layer but realised in lowerlevel embedded-software, resulting in appropriate LoC actuation or sensor excitation. Biosensing data is acquired at the HWA layer and transmitted back to mobile-software, through a decoder object, for analytics, storage, user-interfacing, etc.

derive, fetch and translate the relevant sequences of actuation or acquisition instructions to the LoC instrumentation and control drivers.

When a smart-device is coupled to an instrumented LoC- 


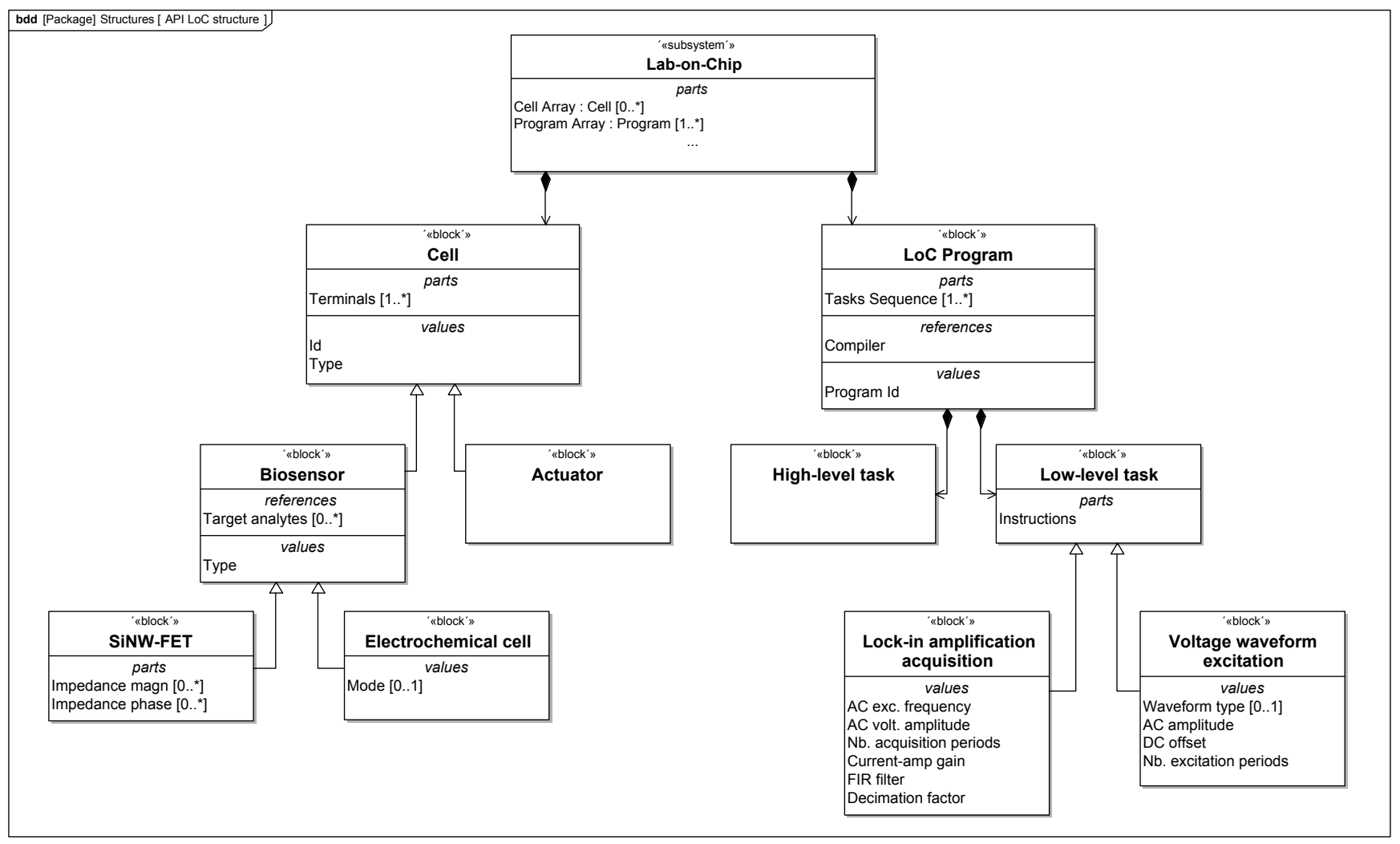

Figure 3. LoC software model: The abstracted LoC is specified according to the architecture and configuration of the specific physical LoC to be interfaced to the HWA. These properties consist of an array of biosensors or actuators of various types, each physically mapped to the I/O terminals of the HWA. The abstracted-LoC also comprehends references to an array of LoC-programs each helping to realise a PoC IVMT use-case. These programs are composed of intertwined low-level routines (performed in the HWA or the LoC, e.g. lock-in amplification acquisition) or high-level tasks (e.g. data analytics) realised in mobile-software or in the cloud.

based system, instrumentation and control functions are most often allocated to a specifically designed hardware accessory (HWA) interfaced to the smart-device via a connector (dongle-accessory) or wirelessly. LoCs are physically interfaced to the HWA during LoC program execution, and later disposed of (single-use LoCs are predominant in IVMT applications to avoid biological fouling and resulting reliability issues). This system architecture predominantly involves the design and execution of the LoC programs at the HWA embedded-software/hardware abstraction layer, leaving the smart-device mostly responsible for higher-level software data analytics and user-interfacing (Fig. 1). While this design may provide several advantages (e.g. real-time system requirements management) it shows limited evolvability. The implementation of new LoC programs will not only require low-level embedded-software/hardware reengineering but also necessarily, to a certain extent, the partial reshaping of higher-level mobile software. The concurrent efforts to redesign both lower- and higher-level system layers will also require engineering team coordination to rethink the different software-layers interfaces and to eventually validate their integration. These activities will cost time and resources, hindering the realisation and further adoption of the new use-case or technology.

Taking into account these considerations, we suggest instead an architecture propagating LoC programability and the LoC instrumentation and control tools to mobile-software, concentrating design and engineering efforts at higherabstraction layers (Fig. 2). We present the design and implementation of such a system capable of interfacing LoCs embedding current- or impedance-based biosensors such as Silicon Nanowire Field Effect Transistors (SiNW-FET) [36] or electrochemical transducers [37]-[40].

\section{A SMARTPHONE-BASED DIAGNOSTICS PLATFORM DESIGNED FOR EVOLVABILITY}

\section{A. System design overview}

The design of our platform involved the abstraction and compartmentalisation of a generic LoC device embedding an arbitrary number of cells (e.g. biosensors or actuators). These are specified by their type and their physical mapping on the LoC (The software model of the LoC is given in 


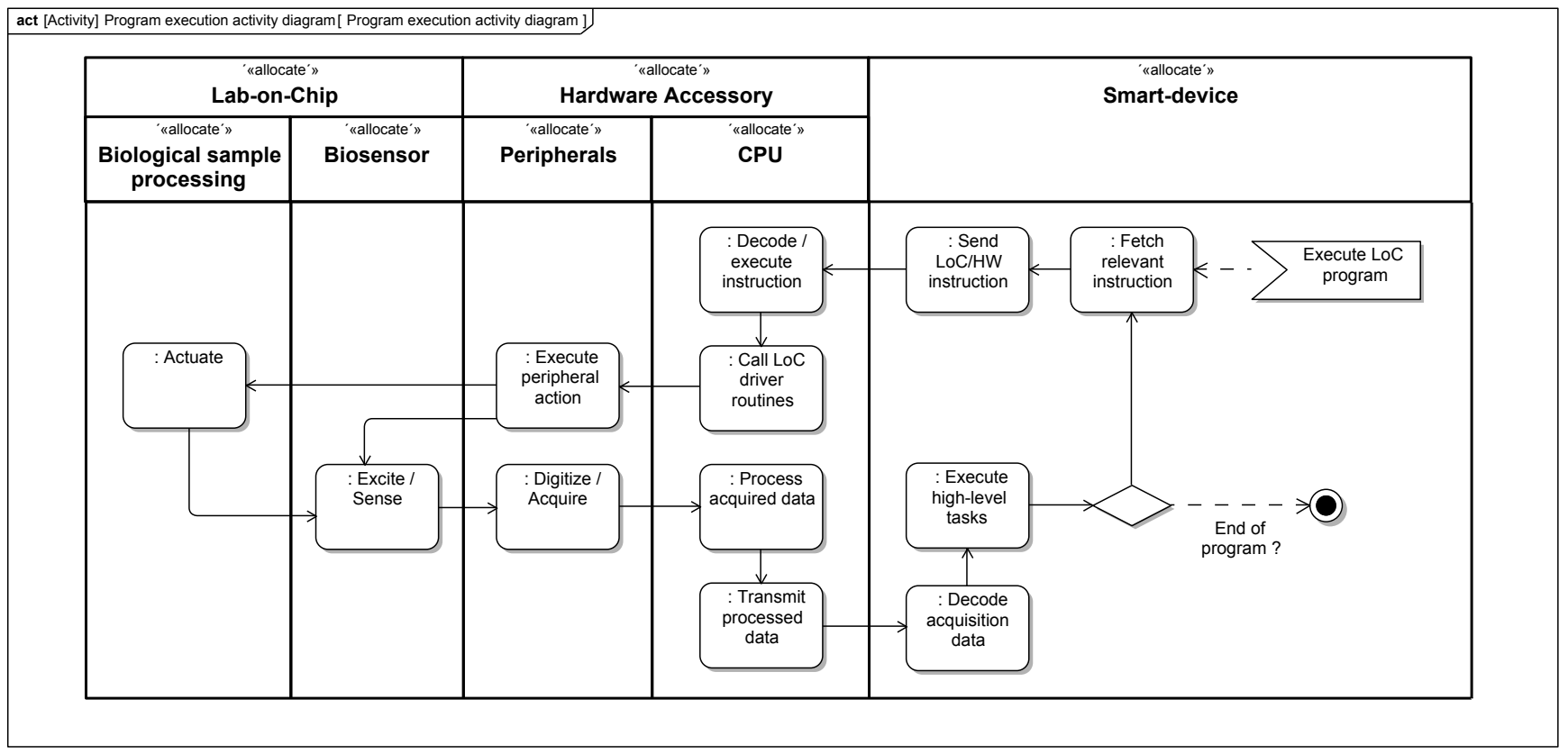

Figure 4. Program execution subsystem allocation - Execution is controlled by the smart-device. Low-level tasks intructions are fetched and transmitted to the HWA which then relays the appropriate sensing or actuation schemes to the LoC. Acquisition data can be preprocessed in the HWA or transmitted directly back to the smart-device for post-processing and analysis. Results from high-level routines performed upon low-level tasks completion can be used in program conditional branching before the next instruction is fetched to the HWA.

Fig. 3). We then determined the instrumentation and control requirements with regards to each possible cell (sensor excitation, acquisitions, actuation, etc.) and used them to objectify drivers in mobile-software. Concurrently, we designed the instrument/reader HWA as well as the interfaces and communication protocols necessary to translate higherlevel mobile-software instructions to downstream embeddedsoftware routines and to enable the upstream transmission of pre-processed or raw acquisition data from the HWA to mobile-software.

\section{B. System architecture}

Our system architecture revolves around an iOS Application Programming Interface (API) providing a highlevel smart-mobile software toolbox for the design of LoC programs.

1) API: The API allows for the pre-compiled or runtime generation of specific LoC programs, adapted to the architecture of the LoC interfaced to the HWA. These LoC programs are executed from the mobile-software layer. An illustration of the software data model for the LoC, some of its possible constituents and their properties is given in Fig. 3.

LoC programs usually consist of low-level tasks, typically acquisition sequences, that rely on the instrumentation and control drivers objectified in iOS. These drivers enable the configuration of voltage waveform excitations, tunable acquisitions of DC currents or the phase-sensitive detection and recovery of $\mathrm{AC}$ currents (i.e. Lock-in amplification [41], [42]). They also allow the programming of specific HWA post-processing routines such as low-pass FIR filtering and decimation. These low-level tasks can then be called on appropriate LoC cells (sensor or actuator) in a timely manner. No actuation driver was considered within the scope of this work but their design and implementation can be envisaged without the need to alter the overall system architecture.

The API also comprehends high-level classes and methods that can virtually consist of any high-level related task: from the call of cloud-computing analytics, to database access, information storage or simple software delays. These highlevel tasks can be intertwined with low-level tasks when composing a LoC program.

Finally LoC program generation and execution handling classes and methods were designed to leverage the aforementioned low- or high-level tasks when designing use-case specific LoC programs. A compiler and a decoder object provide the interfaces necessary to relay LoC-based task instructions and data to or from the HWA.

2) Instrument hardware-accessory: The HWA's architecture revolves around a Central Processing Unit (TMS320F28377D - C2000 Digital Signal Controller, Texas Instruments Inc.). In our case, the HWA CPU embeds the peripherals necessary to achieve tunable voltage wave- 


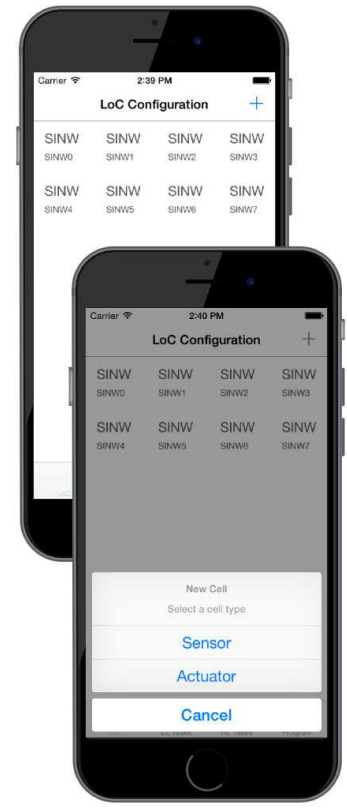

LoC Configuration

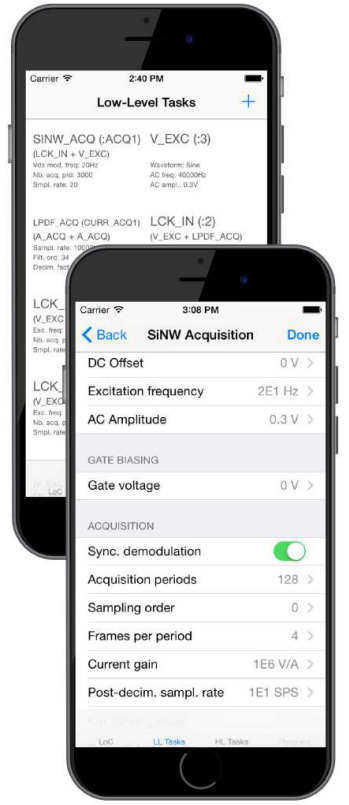

Low-level task customization

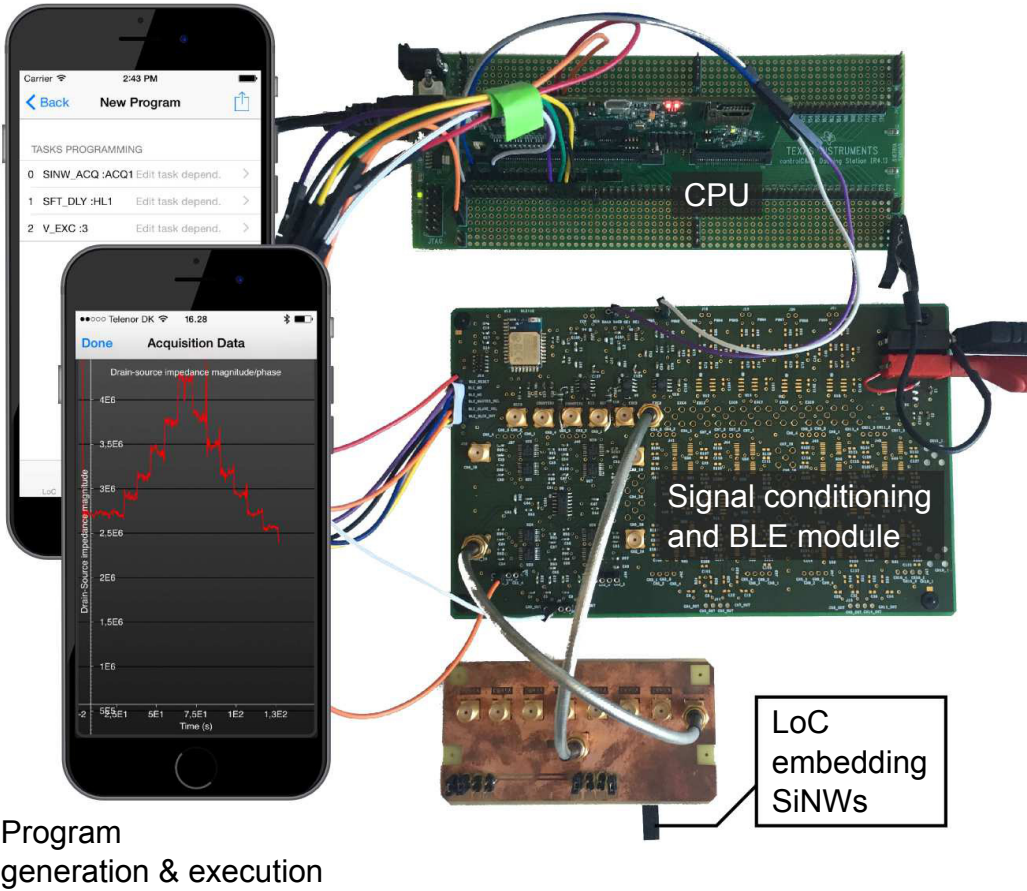

generation \& execution

Figure 5. System hardware setup and iOS mobile application storyboard: From the left: LoC configuration: Define the architecture and configuration of the LoC physically interfaced to the HWA; Low-level tasks definition: Select and customise the low-level routines for acquisitions and/or actuation, map them to the relevant biosensors or actuators previously defined; Program generation: Select either low- or high-level task to define the sequence forming the LoC program, call compilation and execution; Results: Illustration of the lock-in amplification acquisition routine performed on a SiNW-FET at 20Hz, The steps in the impedance magnitude correspond to steps in the electrical potential applied to the gate, simulating target biomarker concentration variations. The HWA materializes at this early development stage by a development board hosting the HWA CPU (TI C2000 TMS320F28377D DSC) interfaced to a break-out PCB embedding the analog signal conditioning circuitry and the BLE module. This PCB is here interfaced to a LoC baring SiNWs. The LoC is plugged to another interface-PCB via a zero-insertion force socket. SMA connectors are wiring the adequate LoC terminals to the relevant HWA I/Os.

forms excitations (12-bit DAC or PWM channels) and multiplexed analog-to-digital conversions (12 multiplexed inputs to ADC modules). Embedded-software routines were implemented to allow for asynchronous or synchronous excitations/acquisitions in order to achieve phase-sensitive detection and the recovery of small AC-signals. The HWA CPU is slave to a Bluetooth Low Energy (BLE) module (BLE112, Bluegiga Inc.) allowing wireless interfacing with iOS. Appropriate signal conditioning circuitry is following the DAC and PWM excitation outputs (reconstruction low-pass filters) or precedes the ADC sampleand-hold circuitry (transimpedance current-amplifiers with programmable gains followed by antialiasing filters). LoC interfacing is, at this early development stage, achieved via coaxial SMA cables adequately connecting the embedded LoC sensors to the chosen HWA I/Os (Fig. 5).

\section{OPERATION}

We designed and implemented a simple iOS mobile application to demonstrate the functionalities of our system prototype. The application user-interface (UI) is built on a tab bar menu. The first tab item presents the embedded-cells (sensors or actuators) specified for the LoC software model.
There, the user should set up the LoC cells so that they reflect the architecture and physical mapping of the specific LoC interfaced to the HWA.

The second tab item allows the configuration of excitations or acquisition sequences along with post-processing routines, mapping them to the relevant cells defined in the previous step. The low-level tasks customisation scene in Fig. 5 presents the configuration of a lock-in amplification acquisition sequence for monitoring both impedance magnitude and phase of a SiNW-FET. These settings include in particular the current-amplifier gain selection, the $\mathrm{AC}$ excitation frequency, the excitation amplitude, the number of acquisition periods, the post-decimation sampling rate and the FIR low-pass filter. Other low-level tasks enable independent customisable current-acquisitions (e.g. for photodiodes sensors), or simple voltage excitations. Classes and methods for electrochemical measurements such as cyclic voltametry, amperometry, square-wave cyclic voltametry or electrochemical impedance spectroscopy have not been implemented but are hardware-ready.

The third tab item presents examples of pre-programmed high-level tasks. While the possibilities there are almost 
unlimited, we implemented simple mobile software delays that can easily be incorporated in a LoC program, for instance those that require latencies between acquisitions. Finally the fourth tab item enables LoC program generation by sequencing either of the previously defined low- or high-level tasks, such as a lock-in amplification acquisition on the first sensor mapped on the LoC, followed by a two-second software delay, followed by a simple sinewave voltage excitation on the third cell mapped on the LoC, etc. Once a LoC program has been configured, a compilation step is necessary before starting execution. The program tasks are then executed sequentially, the completion of data acquisition for one task triggering the next one (Fig. 4). A capture of the drain-source impedance magnitude of a SiNW-FET, recovered by lock-in amplification at $20 \mathrm{~Hz}$ is presented at the right of Fig. 5

\section{Discussion}

The system architecture we propose in this work offers significant system evolvability: The iOS mobile application presented before illustrates the capability of our platform to interface various LoCs embedding a variable number of current- or impedance-based biosensors, potentially targeting different biomarkers. Although the API does not include any actuation drivers at this stage, the available acquisitions schemes make it possible to interface varied passive microfluidic devices, driven by capillary flow. Interfacing these devices will necessitate no or only minor HWA embeddedsoftware upgrades therefore concentrating the development efforts at the mobile-software layer.

Our system architecture also applies for use-cases or LoCs of higher complexity. An example could be that of LoCs embedding their own processing unit: This is becoming increasingly common as the high-level of integration of micro- and nanobiosensors or MEMS often requires the development of complete System-on-Chips (SoC) [43]. This scenario would simply require the design and implementation of additional mobile-software drivers most likely involving the configuration and control of the HWA CPU communication modules (SPI, I2C) without compromising the integrity of the overall system architecture.

The instrumentation and control drivers, coupled to mobilesoftware handling of LoC program execution, enable the retrieval of low-level acquisition data from the execution of the LoC program low-level tasks. This design feature provides significant freedom of action as to how to postprocess the data in higher-level abstraction layers: in mobilesoftware or potentially in the cloud. This property is valuable in a context where -omics sequencing technologies are increasingly coupled to cloud supercomputing analytics [19]. Similarly, the composition of LoC programs intertwining low- and high-level tasks offer significant LoC programmability advantages : high-level tasks return values can be used to modify following LoC program instructions at run time. LoC program conditional branching could thus rely on the evaluation of user-queried or cloud-computing return properties.

Beyond LoC structural or functional modifications, our architecture could also offer significant evolvability advantages should the HWA itself need to be partially or fully redesigned, for instance to accommodate for different actuation or biosensing mechanisms. The overall high-level mobile-software architecture could be preserved to some extent, implying that the modularity of our system may extend not only to the LoCs but to the instrument/reader HWA. This would in turn promise a wide range of applications and usecases all founded on the same high-level software abstraction layer.

Our investigation mainly addressed system evolvability and did not include considerations such as embedded-software real-time system capabilities, system interoperability, regulatory requirements, etc. These elements all contribute to the socio-technical complexity of the PoC IVMT system context and may justify a more systematic and cross-disciplinary approach to system analysis and design, such as ModelBased Systems Engineering (MBSE).

\section{CONCLUSION}

We presented in this work a LoC-based PoC IVMT system architecture designed for evolvability. Relying on LoC programmability and higher-abstraction software layers flexibility, this architecture enabled us to implement a system prototype allowing at this early development stage, the interfacing of various LoCs embedding current- or impedancebased biosensors. Despite the challenges associated with a highly complex socio-technical system context and the current lack of clear LoC-based PoC IVMT system design methodologies, we believe that such a design for evolvability can promote end-user system adoption. We also conjecture that most of the observations and some of the architectural features evoked in this work may not only apply to LoCbased PoC IVMT systems but also to the emerging Lab-ina-Body systems and applications [44]-[46].

\section{ACKNOWLEDGMENT}

This project is a part of the EU Marie Curie Initial Training Networks (ITN) Biomedical engineering for cancer and brain disease diagnosis and therapy development: EngCaBra. Project no. PITN-GA-2010-264417.

\section{REFERENCES}

[1] E. Topol, The Patient Will See You Now: The Future of Medicine is in Your Hands. Basic Books, 2015.

[2] "Global, regional, and national age-sex specific all-cause and cause-specific mortality for 240 causes of death, 1990-2013: a systematic analysis for the global burden of disease study 2013," The Lancet, vol. 385, no. 9963, pp. 117 - 171, 2015. [Online]. Available: http://www.sciencedirect. com/science/article/pii/S0140673614616822 
[3] A. Mills, "Health Care Systems in Low- and Middle-Income Countries," New England Journal of Medicine, vol. 370, no. 6, pp. 552-557, Feb. 2014.

[4] E. Topol, The Creative Destruction of Medicine: How the Digital Revolution Will Create Better Health Care. Basic Books, 2012.

[5] F. S. Collins and H. Varmus, "A new initiative on precision medicine," New England Journal of Medicine, vol. 372, no. 9, pp. 793-795, 2015.

[6] L. Gervais, N. de Rooij, and E. Delamarche, "Microfluidic Chips for Point-of-Care Immunodiagnostics," Advanced Materials, vol. 23, no. 24, pp. H151-H176, Jun. 2011.

[7] C. D. Chin, T. Laksanasopin, Y. K. Cheung, D. Steinmiller, V. Linder, H. Parsa, J. Wang, H. Moore, R. Rouse, G. Umviligihozo, E. Karita, L. Mwambarangwe, S. L. Braunstein, J. van de Wijgert, R. Sahabo, J. E. Justman, W. El-Sadr, and S. K. Sia, "Microfluidics-based diagnostics of infectious diseases in the developing world," Nature Medicine, vol. 17, no. 8, pp. 1015-1019, Jul. 2011.

[8] K. F. Lei, "Microfluidic Systems for Diagnostic Applications: A Review," Journal of Laboratory Automation, vol. 17, no. 5, pp. 330-347, Oct. 2012.

[9] H. Zhu, S. O. Isikman, O. Mudanyali, A. Greenbaum, and A. Ozcan, "Optical imaging techniques for point-of-care diagnostics," Lab Chip, vol. 13, no. 1, pp. 51-67, 2013.

[10] J. Wang, "Electrochemical biosensors: Towards point-of-care cancer diagnostics," Biosensors and Bioelectronics, vol. 21, no. 10, pp. 1887-1892, Apr. 2006.

[11] H. Morgan and D. Spencer, "Chapter 10 microfluidic impedance cytometry for blood cell analysis," in Microfluidics for Medical Applications. The Royal Society of Chemistry, 2015, pp. 213-241. [Online]. Available: http://dx.doi.org/10. 1039/9781849737593-00213

[12] M.-I. Mohammed and M. P. Y. Desmulliez, "Lab-on-a-chip based immunosensor principles and technologies for the detection of cardiac biomarkers: a review," Lab Chip, vol. 11, no. 4, pp. 569-595, 2011.

[13] C. Wyatt Shields IV, C. D. Reyes, and G. P. Lpez, "Microfluidic cell sorting: a review of the advances in the separation of cells from debulking to rare cell isolation," Lab Chip, vol. 15, no. 5, pp. 1230-1249, 2015.

[14] Y. Chen, P. Li, P.-H. Huang, Y. Xie, J. D. Mai, L. Wang, N.-T. Nguyen, and T. J. Huang, "Rare cell isolation and analysis in microfluidics," Lab on a Chip, vol. 14, no. 4, p. 626, 2014.

[15] S. Lindstrm and H. Andersson-Svahn, "Overview of singlecell analyses: microdevices and applications," Lab on a Chip, vol. 10, no. 24, p. 3363, 2010.

[16] Y. Zhao, D. Chen, H. Yue, J. B. French, J. Rufo, S. J. Benkovic, and T. J. Huang, "Lab-on-a-chip technologies for single-molecule studies," Lab on a Chip, vol. 13, no. 12, p. 2183, 2013.

[17] Oxford nanopore minion sequencer. (Accessed: 2015-03-14). [Online]. Available: https://nanoporetech.com/technology/ the-minion-device-a-miniaturised-sensing-system/ the-minion-device-a-miniaturised-sensing-system

[18] E. C. Hayden, "Technology: the \$1,000 genome," Nature, vol. 507, no. 7492, pp. 294-5, 2014.

[19] M. J. Puckelwartz, L. L. Pesce, V. Nelakuditi, L. DellefaveCastillo, J. R. Golbus, S. M. Day, T. P. Cappola, G. W. Dorn, I. T. Foster, and E. M. McNally, "Supercomputing for the parallelization of whole genome analysis," Bioinformatics, vol. 30, no. 11, pp. 1508-1513, Jun. 2014.

[20] M. J. Jebrail, M. S. Bartsch, and K. D. Patel, "Digital microfluidics: a versatile tool for applications in chemistry, biology and medicine," Lab on a Chip, vol. 12, no. 14, p. 2452, 2012.

[21] S.-Y. Teh, R. Lin, L.-H. Hung, and A. P. Lee, "Droplet microfluidics," Lab on a Chip, vol. 8, no. 2, p. 198, 2008.

[22] R. W. Peeling and D. Mabey, "Point-of-care tests for diagnosing infections in the developing world: Point-of-care tests for diagnosing infections," Clinical Microbiology and Infection, vol. 16, no. 8, pp. 1062-1069, Sep. 2009.

[23] B. A, E. DC, H. J, and e. al, "Mobile applications in dermatology," JAMA Dermatology, vol. 149, no. 11, pp. 1300-1304, 2013.

[24] L. Dayer, S. Heldenbrand, P. Anderson, P. O. Gubbins, and B. C. Martin, "Smartphone medication adherence apps: Potential benefits to patients and providers," Journal of the American Pharmacists Association, vol. 53, no. 2, p. 172, Mar. 2013.

[25] B. Li, L. Li, A. Guan, Q. Dong, K. Ruan, R. Hu, and Z. Li, “A smartphone controlled handheld microfluidic liquid handling system," Lab Chip, vol. 14, no. 20, pp. 4085-4092, Aug. 2014.

[26] T. Laksanasopin, T. W. Guo, S. Nayak, A. A. Sridhara, S. Xie, O. O. Olowookere, P. Cadinu, F. Meng, N. H. Chee, J. Kim, C. D. Chin, E. Munyazesa, P. Mugwaneza, A. J. Rai, V. Mugisha, A. R. Castro, D. Steinmiller, V. Linder, J. E. Justman, S. Nsanzimana, and S. K. Sia, "A smartphone dongle for diagnosis of infectious diseases at the point of care," Science Translational Medicine, vol. 7, no. 273, p. 273re1, 2015. [Online]. Available: http://stm.sciencemag.org/content/7/273/273re1.abstract

[27] D. Talbot. (2015, Feb.) A Smartphone Test for HIV and Syphilis Shows Promise in Rwanda. (Accessed: 2015-03-08). [Online]. Available: http://www.technologyreview.com/news/ 534716/smartphone-test-for-hiv-and-syphilis-costs-pennies/

[28] Cue health. (Accessed: 2015-03-16). [Online]. Available: http://www.cuehealth.com

[29] D. Erickson, D. O’Dell, L. Jiang, V. Oncescu, A. Gumus, S. Lee, M. Mancuso, and S. Mehta, "Smartphone technology can be transformative to the deployment of lab-on-chip diagnostics," Lab on a Chip, vol. 14, no. 17, p. 3159, Mar. 2014.

[30] G. Poste, "Bring on the biomarkers," Nature, vol. 469, no. 7329, pp. 156-157, 2011.

[31] W. Koh, W. Pan, C. Gawad, H. C. Fan, G. A. Kerchner, T. Wyss-Coray, Y. J. Blumenfeld, Y. Y. El-Sayed, and S. R. Quake, "Noninvasive in vivo monitoring of tissue-specific global gene expression in humans," Proceedings of the National Academy of Sciences, vol. 111, no. 20, pp. 7361-7366, May 2014

[32] M. Alistar and P. Pop, "Synthesis of biochemical applications on digital microfluidic biochips with operation execution time variability," Integration, the VLSI Journal, Mar. 2015.

[33] J. A. Christian III and J. R. Olds, "A Quantitative Methodology for Identifying Evolvable Space Systems," in 1st AIAA Space Exploration Conference January, 2005.

[34] R. Renaudot, V. Agache, Y. Fouillet, G. Laffite, E. Bisceglia, L. Jalabert, M. Kumemura, D. Collard, and H. Fujita, "A 
programmable and reconfigurable microfluidic chip," Lab on a Chip, vol. 13, no. 23, p. 4517, 2013.

[35] L. M. Fidalgo and S. J. Maerkl, "A software-programmable microfluidic device for automated biology," Lab on a Chip, vol. 11, no. 9, p. 1612, 2011.

[36] G. Zheng, F. Patolsky, and C. Lieber, "Nanowire biosensors: a tool for medicine and life science," Nanomedicine: Nanotechnology, Biology and Medicine, vol. 2, no. 4, p. 277, Dec. 2006.

[37] N. J. Ronkainen, H. B. Halsall, and W. R. Heineman, "Electrochemical biosensors," Chemical Society Reviews, vol. 39, no. 5, p. 1747, 2010.

[38] T. Bakmand, D. Kwasny, M. Dimaki, and W. E. Svendsen, "Fabrication and Characterisation of Membrane-Based Gold Electrodes," Electroanalysis, vol. 27, no. 1, pp. 217-224, Jan. 2015.

[39] M. Dimaki, M. Vergani, A. Heiskanen, D. Kwasny, L. Sasso, M. Carminati, J. Gerrard, J. Emneus, and W. Svendsen, "A Compact Microelectrode Array Chip with Multiple Measuring Sites for Electrochemical Applications," Sensors, vol. 14, no. 6, pp. 9505-9521, May 2014.

[40] F. Alatraktchi, T. Bakmand, M. Dimaki, and W. Svendsen, "Novel Membrane-Based Electrochemical Sensor for RealTime Bio-Applications," Sensors, vol. 14, no. 11, pp. 22 12822 139, Nov. 2014.

[41] R. Burdett, "Amplitude Modulated Signals: The Lock-in Amplifier," Handbook of Measuring System Design, 2005.

[42] N. Li, W. Wang, H. Xu, H. Yu, J. Diao, and D. D.-U. Li, "Wide-Bandwidth Biological Impedance Spectroscopy System Based on the Digital Lock-In Technique," Spectroscopy Letters, vol. 46, no. 7, pp. 476-482, Oct. 2013.

[43] G.-J. Zhang, K. T. C. Chai, H. Z. H. Luo, J. M. Huang, I. G. K. Tay, A. E.-J. Lim, and M. Je, "Multiplexed detection of cardiac biomarkers in serum with nanowire arrays using readout ASIC," Biosensors and Bioelectronics, vol. 35, no. 1, pp. 218-223, May 2012.

[44] A. Yakovlev, D. Pivonka, T. Meng, and A. Poon, "A mmsized wirelessly powered and remotely controlled locomotive implantable device," in Solid-State Circuits Conference Digest of Technical Papers (ISSCC), 2012 IEEE International. IEEE, 2012, pp. 302-304.

[45] T. T. Ruckh and H. A. Clark, "Implantable Nanosensors: Toward Continuous Physiologic Monitoring," Analytical Chemistry, vol. 86, no. 3, pp. 1314-1323, Feb. 2014.

[46] Y. Ling, T. Pong, C. C. Vassiliou, P. L. Huang, and M. J. Cima, "Implantable magnetic relaxation sensors measure cumulative exposure to cardiac biomarkers," Nature Biotechnology, vol. 29, no. 3, pp. 273-277, Mar. 2011. 